\section{Statistical programs for the Apple II microcomputer}

\section{JOSEPH E. STEINMETZ, ANTHONY G. ROMANO, and MICHAEL M. PATTERSON \\ Department of Psychology and College of Osteopathic Medicine Ohio University, Athens, Ohio 45701}

This statistical package contains six analyses of variance, two $t$ tests, and two correlation programs. The ANOVA procedures include one-, two-, and three-way between-subjects analyses, a randomized-block analysis, and two of Kirk's (1968) split-plot factorial (SPF.P.Q and SPF-PR.Q) analyses. Pearson's $r$ and Spearman's rho correlation calculations, as well as independent and correlated $t$ tests, are also included in the package. Computational procedures used in the above programs follow those presented by Bruning and Kintz (1968) or Kirk (1968). ANOVAs involving unequal cell sizes are computed by unweighted means analyses that are available for the two- and three-way between-subjects SPF-P.Q and SPF-PR.Q programs. In addition, a missingobservation estimation routine is supplied for the randomized-block analysis. As currently designed, the length of the largest program (SPF-PR.Q) is approximately $9 \mathrm{~K}$ bytes. Additional memory requirements depend on number of subjects and total number of treatment levels included in the analysis.

Input. The user first selects a statistical routine from a menu that displays the available programs. Once a particular program is selected, various options available for that program are displayed and a series of questions concerning analysis parameters is presented (e.g., NUMBER OF LEVELS OF A?). Among these questions is one asking the user if cell sizes are equal. A negative reply causes an unweighted means solution to be computed.

Raw data points can be entered into the computer by two methods. First, data entries can be directly typed into the computer via the keyboard. When this method is employed, the computer directs the user with specific statements concerning the order of data entry (e.g., ENTER SCORES FOR A1, B1:). If the data have been previously stored in a sequential text file, the file name can be entered and the computer is directed to read the raw data points from disk. Both methods enter data points by cell when between-subjects programs are being used and by subject when within-subjects programs are requested. Subsequent to data entry, data points can be displayed on the monitor for verification. If errors in data entry are detected, they can be corrected through a routine that changes values in the raw data array.

Output. After the computations have been performed, the user has the option to send the output to a printer or to display the results on the monitor. Standard outputs for the ANOVA programs include (1) a summary table with sources of variance, sums of squares, degrees of freedom, mean squares, and $F$ ratios, (2) a table of cell means, (3) a table of marginal means, and (4) an optional listing of data points used in the analysis. The $t$ test program outputs the value of the $t$ ratio, degrees of freedom, and group means, while the correlation program outputs a Pearson's or Spearman's correlation coefficient value. After the computational output has been presented, the user has the option to store the raw data points on disk in the form of a sequential text file. This option allows the user to maintain standard files of research data for future use.

Limitations. Certain restrictions concerning the number of subjects per cell are present. These limitations depend on several factors, including number of treatment levels present in the analysis. As the number of treatment levels increases, the number of subjects that can occupy a cell decreases. The current version of the program creates data array dimensions according to number of levels of the variables that are entered during initial phases of the program. The number of subjects per cell, however, has been fixed at 30 . If overall memory capabilities permit, the maximum number of subjects per cell can easily be changed by altering data array dimension statements that appear at the beginning of the program. Information concerning these changes is provided in program documentation.

Language and Computer. The programs are written in APPLESOFT BASIC and have been implemented on an Apple II (plus) microcomputer with $48 \mathrm{~K}$ of memory. This particular form of BASIC contains some nonstandard commands and procedures that can easily be converted to other forms of BASIC. The programs currently reside on a standard 5.25-in. 16-sector diskette that, when booted automatically, displays a selection menu of available programs.

Availability. Listing and documentation concerning this package are available without charge from Michael M. Patterson, Director of Research Affairs, College of Osteopathic Medicine, Ohio University, Athens, Ohio 45701. Individuals who desire a copy of the package diskette should send an unformatted 5.25-in. floppy disk on which the programs can be duplicated. Work is currently underway to expand this set of programs to include other factorial designs (e.g., analysis of covariance). These additional programs will be included in the package when completed.

\section{REFERENCES}

Bruning, J. L., \& Kintz, B. L. Computational handbook of statistics. Glenview, Ill: Scott, Foresman, 1968.

Kırx, R. E. Experimental design: Procedures for the behavioral sciences. Belmont, Calif: Wadsworth, 1968.

(Accepted for publication September 22, 1981.) 